Supporting Information

\title{
Towards Carbon-Neutral Electric Power Systems in New York State: A Novel Multi-Scale Bottom-Up Optimization Framework Coupled with Machine Learning for Capacity Planning at Hourly Resolution
}

\author{
Ning Zhao ${ }^{1}$, Fengqi You ${ }^{1,2, *}$ \\ ${ }^{1}$ Systems Engineering, Cornell University, Ithaca, New York, 14853, USA \\ ${ }^{2}$ Robert Frederick Smith School of Chemical and Biomolecular Engineering, Cornell University, \\ Ithaca, New York, 14853, USA
}

Wednesday, December 8, 2021

Submitted to ACS Sustainable Chemistry \& Engineering

Number of Pages: 14

Number of Figures: 0

Number of Tables: 0

* Corresponding author. Phone: (607) 255-1162; Fax: (607) 255-9166; E-mail: fengqi.you@cornell.edu 


\section{Mathematical optimization model formulation}

\section{Objective function and economic constraints}

The objective function of minimizing the total electricity transition cost is presented in (1), accounting for the net present cost for capacity planning $(t c d)$ and the net present cost for systems operations (tco) over the planning horizon. The net present costs for capacity planning and systems operations can be calculated through eqs (2) and (3), respectively. $T_{d e s}$ and $T_{\text {opt }}$ represent the sets of design years and operational years, respectively, and TINI is the initial year of the planning horizon. Set $I$ denotes the set of all generation and energy storage technologies, while set $S$ consists of neighbor regions, from which electricity can be imported. $c d e s_{i, t}$ refers to the capacity planning cost of generators or storage units using technology $i$ in year $t$, copt $t_{i, t}$ denotes the operational cost of technology $i$ in year $t$, and $\operatorname{cimp}_{s, t}$ is the electricity importation cost from source $s$ in year $t$. Parameter RATE is the discount rate.

$$
\begin{aligned}
& \min t c d+t c o \\
& t c d=\sum_{t \in T_{d e s}-\{T I N I\}}\left(\frac{\sum_{i \in I} c d e s_{i, t}}{(1+R A T E)^{t-T I N I-1}}\right) \\
& t c o=\sum_{t \in T_{\text {opt }}}\left(\frac{\sum_{i \in I} c o p t_{i, t}+\sum_{s \in S} c i m p_{s, t}}{(1+R A T E)^{t-T I N I-1}}\right)
\end{aligned}
$$

Eqs (4) and (5) calculate the capacity planning costs for generators and the power storage facilities, respectively. The capacity planning costs include the capital costs and the decommissioning costs. $Y$ is the set of ages for generators and energy storage units, and the age of new units is $\mathrm{y}=0$. Set $K$ consists of a list of identification numbers to differentiate units with the same technology and same age in a year. Sets $I_{e}$ and $I_{b}$ denote the set of generation technologies and power storage technologies, respectively.

$$
\begin{aligned}
c d e s_{i, t} & =\sum_{k \in K} \sum_{y \in Y}\left(C C A P_{i, t, y} \cdot e e x t_{i, t, y, k}+\left(C S A L_{i, t}+C D E P_{i, t, y}\right) \cdot e d i s_{i, t, y, k}\right) \\
& , \forall i \in I_{e}, t \in T_{d e s}-\{T I N I\} \\
c d e s_{i, t} & =\sum_{y \in Y}\left(C C A P_{i, t, y} \cdot \operatorname{bext}_{i, t, y}+\left(C S A L_{i, t}+C D E P_{i, t, y}\right) \cdot b d i s_{i, t, y}\right) \\
& , \forall i \in I_{b}, t \in T_{d e s}-\{T I N I\}
\end{aligned}
$$

In terms of the capital costs, $C C A P_{i, t, y}$ represents the unit capital cost for constructing a new 
unit of technology $\mathrm{i}$ in year $\mathrm{t}$, which equals zero when $\mathrm{y} \neq 0$. eext $t_{i, t, y, k}$ is the electricity generation capacity from generator $k$ with technology $i$ existing in period $\mathrm{t}$ with an age of $y$, while bexti,ty denotes the electricity storage capacity of technology $i$ existing in year $t$ with an age of $y$. As for the retirement costs, $C S A L_{i, t}$ is the unit decommissioning cost for retired facility $k$ using technology $i$ in year $t$ at the end of its useful life, while $C D E P_{i, t, y}$ represents the cost due to early retirement for units using technology $i$ in year $t$ with an age of $y$. edis $s_{i, t, y, k}$ is the electricity generation capacity of generator $k$ with technology $i$ decommissioned in period $t$ with an age of $y$, and $b d i s_{i, t, y}$ is the retired energy storage capacity of technology $i$ in year $t$ with an age of $y$.

The operational cost for electricity generation is shown in (6), including the fixed and variable operational costs. The first term on the right-hand side stands for fixed operational cost, where CFIX $X_{i, t, y}$ is the fixed operations and maintenance (O\&M) cost on unit generation capacity using technology $i$ in year $t$ with an age of $y$. The second term refers to the variable O\&M cost, where set $T_{r e p}$ is the set of representative hours in a year, egen ${ }_{i, t, y, k, t}$ is the electricity generated at hour $t t$ from unit $k$ with technology $i$ existing in year $t$ with an age of $y$, and $C V A R_{i, t, y}$ is the unit variable O\&M cost using technology $i$ in year $t$ with an age of $y$.

$$
\begin{aligned}
\operatorname{copt}_{i, t}= & \sum_{y \in Y} \sum_{k \in K} C F I X_{i, t, y} \cdot \operatorname{eext}_{i, t, y, k} \\
& +\sum_{y \in Y} \sum_{k \in K} \sum_{t t \in T_{e p}} \operatorname{egen}_{i, t, y, k, t t} \cdot\left(C V A R_{i, t, y, t t}+E E_{i, t, y} \cdot C C A R_{t}\right) \cdot \frac{E^{\prime}}{R E P N_{t}} \\
& , \forall i \in I_{e}, t \in T_{o p t}
\end{aligned}
$$

where $E E_{i, t, y}$ denotes the greenhouse gas emissions from unit amount of electricity output using technology $i$ in year $t$ with an age of $y$, and $C C A R_{t}$ is the estimated social cost for carbon dioxide emissions in year $t . E A N N_{t}$ and $R E P E_{t}$ stand for the annual electricity demand in year $t$ and the total electricity demand from the representative days of year $t$, respectively.

The O\&M costs for energy storage units can be calculated by eq (7), where parameter $C O P T_{i, t, y}$ denotes the annual O\&M cost for storage technology $i$ in year $t$ with an age of $y$.

$$
\operatorname{copt}_{i, t}=\sum_{y \in Y} C O P T_{i, t, y} \cdot \text { bext }_{i, t, y}, \forall i \in I_{b}, t \in T_{o p t}
$$

$\mathrm{Eq}(8)$ stands for the power importation costs, where $C I M P_{s, t, t t}$ is the unit price of electricity imported from source $s$ in year $t$ at hour $t$, and eimps,t,tt represents the hourly importation of electricity at hour $t t$ in year $y$ from source $s$. 


$$
\operatorname{cimp}_{s, t}=\sum_{t t \in T_{\text {rep }}} C I M P_{s, t, t t} \cdot \operatorname{eimp}_{s, t, t t} \cdot \frac{E A N N_{t}}{R E P E_{t}}, \forall s \in S, t \in T_{\text {opt }}
$$

\section{Energy demand and electricity storage balance constraints}

The hourly electricity demands are depicted by constraint (9), which considers electricity generation, power importation and exportation, and the charging and discharging activities of energy storage facilities. The hourly power demand in hour $t t$ of year $t$ is denoted as $D E M E_{t, t t}$.

$$
\begin{aligned}
\sum_{i \in I_{e}} \sum_{y \in Y} \sum_{k \in K} \operatorname{egen}_{i, t, y, k, t t} & +\sum_{s \in S}\left(\operatorname{eimp}_{s, t, t t}-\operatorname{eexp}_{s, t, t t}\right)+\sum_{i \in I_{b}} \sum_{y \in Y}\left(\text { bout }_{i, t, y, t t}-\operatorname{bin}_{i, t, y, t t}\right) \\
& =D E M E_{t, t t}, \forall t \in T_{\text {opt }}, t t \in T_{\text {rep }}
\end{aligned}
$$

where $\operatorname{eexp}_{s, t, t t}$ stands for the hourly power exportation at hour $t t$ in year $y$ to destination $s$. bout $t_{i, t, y, t t}$ and $\operatorname{bin}_{i, t, y, t t}$ denotes the discharging and charging amounts of electricity for energy storage facilities at hour $t t$ using technology $i$ in year $t$ with an age of $y$, respectively.

Constraints (10) and (11) represent the hourly electricity storage balances for operational periods. bact $_{i, t, y, t}$ is the energy storage level at hour $t t$ from technology $i$ existing in year $t$ with an age of $y$. HINI and HMAX refer to the first hour and the last hour of the representative hours in a year, respectively. Parameter $B L O S_{i, t, y}$ depicts the hourly energy loss for power storage technology $i$ in year $t$ with an age of $y$. Note that a "cyclic" manner of power storage level is applied to maintain the energy balance for each storage facility in each operational year.

$$
\begin{aligned}
& \text { bact }_{i, t, y, t t}=\left(1-\text { BLOS }_{i, t, y}\right) \cdot \text { bact }_{i, t, y, t t-1}-\text { bout }_{i, t, y, t t}+\text { bin }_{i, t, y, t t} \\
&, \forall i \in I_{b}, t \in T_{o p t}, y \in Y, t t \in T_{r e p}-\{H I N I\} \\
& \text { bact }_{i, t, y, t t=H I N I}=\left(1-\text { BLOS }_{i, t, y}\right) \cdot \text { bact }_{i, t, y, t t=H M A X}-\text { bout }_{i, t, y, t t=H I N I}+\operatorname{bin}_{i, t, y, t t=H I N I} \\
&, \forall i \in I_{b}, t \in T_{\text {opt }}, y \in Y
\end{aligned}
$$

\section{Energy availability constraints}

Constraint (12) stands for the maximum hourly power generation from non-intermittent energy sources. Set $I_{e n i}$ consists of all generation technologies that use no intermittent energy.

$$
\text { egen }_{i, t, y, t t, k} \leq \text { eext }_{i, t, y, k}, \forall i \in I_{\text {eni }}, t \in T_{\text {opt }}, y \in Y, t t \in T_{\text {rep }}, k \in K
$$

The maximum hourly electricity outputs from intermittent energy sources, namely wind and solar, are shown in constraint (13). Set $I_{e i}$ includes power generation technologies based on wind 
and solar energy. $C F_{i, t, y, t, k}$ denotes the hourly fractional availability of rated power capacities based on intermittent energy sources.

$$
\text { egen }_{i, t, y, t t, k} \leq C F_{i, t, y, t t} \cdot \text { eext }_{i, t, y, k}, \forall i \in I_{e i}, t \in T_{\text {opt }}, y \in Y, t t \in T_{r e p}, k \in K
$$

The maximum energy storage levels are depicted by constraint (14), where $B F_{i, t, y}$ is the energy storage factor regarding unit storage capacity using technology $i$ in year $t$ with an age of $y$.

$$
\text { bact }_{i, t, y, t t} \leq B F_{i, t, y} \cdot \text { bext }_{i, t, y}, \forall i \in I_{b}, t \in T_{o p t}, y \in Y, t t \in T_{\text {rep }}
$$

Constraints (15) and (16) stand for the hourly charging and discharging limits for electricity storage facilities, respectively. In other words, the hourly energy changes of a storage unit should be less than or equal to its capacity.

$$
\begin{aligned}
& \operatorname{bin}_{i, t, y, t t} \leq \operatorname{bext}_{i, t, y}, \forall i \in I_{b}, t \in T_{\text {opt }}, y \in Y, t t \in T_{\text {rep }} \\
& \text { bout }_{i, t, y, t t} \leq \operatorname{bext}_{i, t, y}, \forall i \in I_{b}, t \in T_{\text {opt }}, y \in Y, t t \in T_{\text {rep }}
\end{aligned}
$$

The hourly power importation and exportation limits are shown in constraint (17) and (18), respectively, where $I M P M_{s, t}$ denotes the maximum electricity transmission capacity between neighbor region $s$ and the region of interest in year $t$.

$$
\begin{aligned}
& \operatorname{eimp}_{s, t, t t} \leq I M P M_{s, t}, \forall s \in S, t \in T_{\text {opt }}, t t \in T_{r e p} \\
& \operatorname{eexp}_{s, t, t t} \leq I M P M_{s, t}, \forall s \in S, t \in T_{\text {opt }}, t t \in T_{r e p}
\end{aligned}
$$

\section{Power systems scheduling constraints}

Scheduled power systems capacity changes are incorporated in the proposed framework to obtain more realistic and accurate electricity transition pathways, including the scheduled power generator additions, scheduled generator deactivations, and the proposed installations of storage units. It is essential to include these scheduled changes, as significant changes to the electric power systems may have been scheduled.

Constraints (19) and (20) depict the scheduled additions of generators and electricity storage facilities, respectively. $P R O G_{i, t, k}$ denotes the scheduled generation capacity additions of generator $k$ with technology $i$ in year $t$, and $P R O B_{i, t, k}$ accounts for the proposed energy storage capacity additions using technology $i$ in year $t$.

$$
\begin{aligned}
& \operatorname{eext}_{i, t, y=0, k} \geq \operatorname{PROG}_{i, t, k}, \forall i \in I_{e}, t \in T_{d e s}, k \in K \\
& \text { bext }_{i, t, y=0} \geq P R O B_{i, t}, \forall i \in I_{b}, t \in T_{d e s}
\end{aligned}
$$


Constraint (21) represents the scheduled deactivations of generators. $P R O D_{i, t, y, k}$ is the proposed decommissioning capacity of generator $k$ that uses technology $i$ in year $t$ with an age of $y$.

$$
e d i s_{i, t, y, k} \geq P R O D_{i, t, y, k}, \forall i \in I_{e}, t \in T_{d e s}, y \in Y, k \in K
$$

\section{Energy and emissions target constraints}

The energy and emissions target constraints include the limits on annual greenhouse gas emissions, the requirements on renewable portfolio standards, and the capacity requirements for renewable generation technologies and energy storage units.

The limits on annual greenhouse gas emissions for the electric power sector are incorporated by constraint (22), where $E L M T_{t}$ denotes the limit for annual greenhouse gas emission in year $t$.

$$
\sum_{i \in I_{e}} \sum_{y \in Y} \sum_{k \in K} \sum_{t t \in T_{\text {rep }}} \operatorname{egen}_{i, t, y, k, t t} \cdot E E_{i, t, y} \cdot \frac{E A N N_{t}}{R E P E_{t}} \leq E L M T_{t}, \forall t \in T_{o p t}
$$

Constraint (23) represents the requirements on renewable portfolio standards proposed by the government. Set $I_{e r}$ consists of generation technologies that use renewable energy sources, and $R P S G_{t}$ is the renewable portfolio standard for these technologies in year $t$.

$$
\sum_{i \in I_{\text {er }}} \sum_{y \in Y} \sum_{k \in K} \sum_{t t \in T_{\text {rep }}} \operatorname{egen}_{i, t, y, k, t t} \geq R P S G_{t} \cdot \sum_{i \in I_{e}} \sum_{y \in Y} \sum_{k \in K} \sum_{t t \in T_{\text {rep }}} \operatorname{egen}_{i, t, y, k, t t}, \forall t \in T_{\text {opt }}
$$

The capacity requirements of renewable generators and energy storage facilities are respectively presented by constraints (24) and (25). $T A R_{i, t}$ represents the minimum generation capacity using technology $i$ in year $t$, while $T A R S_{t}$ denotes the target capacity level for energy storage in year $t$.

$$
\begin{aligned}
& \sum_{y \in Y} \sum_{k \in K} \operatorname{eext}_{i, t, y, k} \geq T A R_{i, t}, \forall i \in I_{e}, t \in T_{o p t} \\
& \sum_{i \in I_{b}} \sum_{y \in Y} \operatorname{bext}_{i, t, y} \geq T A R S_{t}, \forall t \in T_{o p t}
\end{aligned}
$$

\section{Transition relationship constraints}

Constraints (26) and (27) depict the initialization of capacity variables for generators and electricity storage units. Parameters $I N I E_{i, y, k}$ denotes the electricity generation capacity for generator $k$ using technology $i$ with an age of $y$ in the initial year TINI, and $I N I B_{i, y}$ is the energy 
storage capacity using technology $i$ with an age of $y$ in the initial year.

$$
\begin{aligned}
& \operatorname{eext}_{i, t=T I N I, y, k}=\operatorname{INIE}_{i, y, k}, \forall i \in I_{e}, y \in Y, k \in K \\
& \operatorname{bext}_{i, t=T I N I, y}=I N I B_{i, y}, \forall i \in I_{b}, y \in Y
\end{aligned}
$$

Transition relationships for generation capacities across the years within the planning horizon are represented by constraint (28). Specifically, the generation capacity of generator $k$ using technology $i$ in year $t$ with an age $y$ equals the capacity of this generator in the last year subtracted by the corresponding deactivated capacity. Sets $T_{\text {year }}$ and $T_{d e s}$ stand for all years and design years within the planning horizon, respectively. The generator installation or deactivation decisions, namely the capacity planning decisions, are made in the design years within $T_{\text {des }}$.

$$
\operatorname{eext}_{i, t, y, k}=\operatorname{eext}_{i, t-1, y-1, k}-e d i s_{i, t, y, k}, \forall i \in I_{e}, t \in T_{\text {year }}-\{T I N I\}, y \geq 1, k \in K
$$

Similarly, the transition relationships for energy storage capacities are depicted by constraint (29).

$$
\text { bext }_{i, t, y}=\text { bext }_{i, t-1, y-1}-\text { bdis }_{i, t, y}, \forall i \in I_{b}, t \in T_{\text {year }}-\{T I N I\}, y \geq 1
$$

The generators and the energy storage units are assumed to be deactivated if their ages exceed the corresponding lifetime, as represented by constraints (30) and (31). LIFE $E_{i}$ stands for the lifetime of a generator or storage unit using technology $i$.

$$
\begin{aligned}
& \text { edis }_{i, t, y, k}=\operatorname{eext}_{i, t-1, y-1, k}, \forall i \in I_{e}, t \in T_{\text {des }}-\{T I N I\}, y \geq \text { LIFE }_{i}, k \in K \\
& \text { bdis }_{i, t, y}=\text { bext }_{i, t-1, y-1}, \forall i \in I_{b}, t \in T_{\text {des }}-\{T I N I\}, y \geq \text { LIFE }_{i}
\end{aligned}
$$

Constraint (32) limits the maximum capacity of a new generator during the energy transition, where $L C A P_{i, t, y}$ is the capacity limit for new generator $k$ using technology $i$ in year $t$.

$$
\operatorname{eext}_{i, t, y=0, k} \leq L C A P_{i, t, y=0}, \forall i \in I_{e}, t \in T_{\text {des }}-\{T I N I\}, k \in K
$$

The decommissioning of a generator can be depicted by constraints (33) and (34). Specifically, binary variable $u_{i, t, y, k}$ indicates whether generator $k$ with technology $i$ in year $t$ with an age of $y$ is to be deactivated or not. $M$ and $\varepsilon$ represent a large positive number and a small positive number, respectively.

$$
\begin{aligned}
& \epsilon \cdot u_{i, t, y, k} \leq e \operatorname{edis}_{i, t, y, k} \leq M \cdot u_{i, t, y, k}, \forall i \in I_{e}, t \in T_{d e s}-\{\text { TINI }\}, y \in Y, k \in K \\
& \operatorname{edis}_{i, t, y, k}=\operatorname{eext}_{i, t-1, y-1, k} \cdot u_{i, t, y, k}, \forall i \in I_{e}, t \in T_{d e s}-\{T I N I\}, y \in Y, k \in K
\end{aligned}
$$

Note that constraint (34) has a bilinear structure, and Glovers linearization is applied to reformulate the bilinear constraint into multiple linear ones to facilitate the solution process. The 
reformulated constraints are presented in constraints (35)-(37), where $L B C_{i}$ and $U B C_{i}$ denote the lower and upper bounds of a new generator using technology $i$.

$$
\begin{aligned}
& L B C_{i} \cdot u_{i, t, y, k} \leq \operatorname{edis}_{i, t, y, k} \leq U B C_{i} \cdot u_{i, t, y, k}, \forall i \in I_{e}, t \in T_{d e s}-\{T I N I\}, y \in Y, k \in K \\
& \text { edis }_{i, t, y, k} \geq \operatorname{eext}_{i, t-1, y-1, k}-U B C_{i} \cdot\left(1-u_{i, t, y, k}\right), \forall i \in I_{e}, t \in T_{d e s}-\{T I N I\}, y \in Y, k \in K \\
& \text { edis }_{i, t, y, k} \leq \operatorname{eext}_{i, t-1, y-1, k}-L B C_{i} \cdot\left(1-u_{i, t, y, k}\right), \forall i \in I_{e}, t \in T_{d e s}-\{T I N I\}, y \in Y, k \in K
\end{aligned}
$$

\section{Transition capacity constraints}

Constraints (38) and (39) represent the maximum capacity increase limits for generators and electricity storage units, respectively, where $G R O E_{i, t}$ is the maximum generation capacity increase using technology $i$ in year $t$, and $G R O B_{i, t}$ stands for the maximum increase of electricity storage capacity of technology $i$ in year $t$.

$$
\begin{aligned}
& \sum_{y \in Y} \sum_{k \in K} \operatorname{eext}_{i, t, y, k}-\sum_{y \in Y} \sum_{k \in K} \operatorname{eext}_{i, t-1, y, k} \leq G R O E_{i, t}, \forall i \in I_{e}, t \in T_{d e s}-\{T I N I\} \\
& \sum_{y \in Y} \operatorname{bext}_{i, t, y}-\sum_{y \in Y} \operatorname{bext}_{i, t-1, y} \leq G R O B_{i, t}, \forall i \in I_{b}, t \in T_{d e s}-\{T I N I\}
\end{aligned}
$$

Similarly, the capacity decrease limits for generators and electricity storage units are depicted by constraints (40) and (41). $R E D E_{i, t}$ and $R E D B_{i, t}$ denotes the maximum capacity reduction corresponding to technology $i$ in year $t$ for the generators and energy storage units, respectively.

$$
\begin{aligned}
& \sum_{y \in Y} \sum_{k \in K} \operatorname{eext}_{i, t-1, y, k}-\sum_{y \in Y} \sum_{k \in K} \operatorname{eext}_{i, t, y, k} \leq R E D E_{i, t}, \forall i \in I_{e}, t \in T_{d e s}-\{T I N I\} \\
& \sum_{y \in Y} \operatorname{bext}_{i, t, y}-\sum_{y \in Y} \operatorname{bext}_{i, t-1, y} \leq R E D B_{i, t}, \forall i \in I_{b}, t \in T_{d e s}-\{T I N I\}
\end{aligned}
$$

The total generation capacity changes across different planning years are presented in constraints (42) and (43), where $T R E_{t}$ stands for the total generation capacity changes in percentage.

$$
\begin{aligned}
& \sum_{i \in I_{e}} \sum_{y \in Y} \sum_{k \in K} \operatorname{eext}_{i, t, y, k} \geq\left(1-\text { TRE }_{t}\right) \cdot \sum_{i \in I_{e}} \sum_{y \in Y} \sum_{k \in K} \operatorname{eext}_{i, t-1, y, k}, \forall t \in T_{d e s}-\{T I N I\} \\
& \sum_{i \in I_{e}} \sum_{y \in Y} \sum_{k \in K} \operatorname{eext}_{i, t, y, k} \leq\left(1+T R E_{t}\right) \cdot \sum_{i \in I_{e}} \sum_{y \in Y} \sum_{k \in K} \operatorname{eext}_{i, t-1, y, k}, \forall t \in T_{d e s}-\{T I N I\}
\end{aligned}
$$

Constraints (44) and (45) show the total capacity changes for electricity storage between consecutive years within the planning horizon. $T R B_{t}$ denotes the total energy storage capacity changes in percentage. 


$$
\begin{aligned}
& \sum_{i \in I_{b}} \sum_{y \in Y} \sum_{k \in K} \operatorname{bext}_{i, t, y, k} \geq\left(1-T R B_{t}\right) \cdot \sum_{i \in I_{b}} \sum_{y \in Y} \sum_{k \in K} \operatorname{bext}_{i, t-1, y, k}, \forall t \in T_{d e s}-\{T I N I\} \\
& \sum_{i \in I_{b}} \sum_{y \in Y} \sum_{k \in K} \operatorname{bext}_{i, t, y, k} \leq\left(1+T R B_{t}\right) \cdot \sum_{i \in I_{b}} \sum_{y \in Y} \sum_{k \in K} \operatorname{bext}_{i, t-1, y, k}, \forall t \in T_{d e s}-\{T I N I\}
\end{aligned}
$$

\section{Problem size reduction constraints}

To further alleviate the computational burden, status indicators are introduced to keep the capacity and operational variables for potentially active generation and energy storage units, while they can remove the redundant variables associated with unrealistic combinations of technology, capacity, and age for a certain unit. The status indicators $\left(B I N D_{i, t, y}\right)$ for active electricity storage facilities are presented in eqs (46)-(48).

$$
\begin{aligned}
& B I N D_{i, t=T I N I, y}=\left\{\begin{array}{l}
1, \forall i \in I_{b}, y \in Y, I N I B_{i, y}>0 \\
0, \forall i \in I_{b}, y \in Y, I N I B_{i, y}=0
\end{array}\right. \\
& B I N D_{i, t, y=0}=1, \forall i \in I_{b}, t \in T_{d e s}-\{T I N I\} \\
& B I N D_{i, t, y}=1, \forall i \in I_{b}, t \in T_{d e s}-\{T I N I\}, 1 \leq y \leq \text { LIFE }_{i}, B I N D_{i, t-1, y-1}=1
\end{aligned}
$$

The active status indicators $\left(E I N D_{i, t, y, k}\right)$ for generators can be calculated by eqs (49)-(51).

$$
\begin{aligned}
& \operatorname{EIND}_{i, t=T I N I, y, k}=\left\{\begin{array}{l}
1, \forall i \in I_{e}, y \in Y, k \in K, I N I E_{i, y, k}>0 \\
0, \forall i \in I_{e}, y \in Y, k \in K,{I N I E_{i, y, k}}=0
\end{array}\right. \\
& \text { EIND }_{i, t, y=0, k}=1, \forall i \in I_{e}, t \in T_{d e s}-\{T I N I\}, k \in K \\
& \text { EIND }_{i, t, y, k}=1, \forall i \in I_{e}, t \in T_{\text {des }}-\{T I N I\}, 1 \leq y \leq \text { LIFE }_{i}, k \in K, \text { EIND }_{i, t-1, y-1, k}=1
\end{aligned}
$$

The application of status indicators can significantly reduce the number of redundant variables

for both capacity planning and systems operations, allowing the framework to incorporate the additional age information for each unit that is usually omitted in the existing literature. The problem size reduction constraints for storage-related decision variables are shown in constraints (52)-(56).

$$
\begin{aligned}
& \text { bact }_{i, t, y, t t}=0, \forall i \in I_{b}, t \in T_{o p t}, y \in Y, t t \in T_{r e p}, B I N D_{i, t, y}=0 \\
& \text { bin }_{i, t, y, t t}=0, \forall i \in I_{b}, t \in T_{o p t}, y \in Y, t t \in T_{r e p}, B I N D_{i, t, y}=0 \\
& \text { bout }_{i, t, y, t t}=0, \forall i \in I_{b}, t \in T_{o p t}, y \in Y, t t \in T_{r e p}, B I N D_{i, t, y}=0 \\
& \text { bext }_{i, t, y}=0, \forall i \in I_{b}, t \in T_{d e s}, y \in Y, B I N D_{i, t, y}=0
\end{aligned}
$$




$$
b_{d i s_{i, t, y}}=0, \forall i \in I_{b}, t \in T_{d e s}, y \in Y, B I N D_{i, t, y}=0
$$

Similarly, constraints (57)-(61) represent the size-reduction constraints for the decision variables related to power generation.

$$
\begin{aligned}
& \operatorname{egen}_{i, t, y, k, t t}=0, \forall i \in I_{e}, t \in T_{o p t}, y \in Y, k \in K, t t \in T_{r e p}, \operatorname{EIND}_{i, t, y, k}=0 \\
& \operatorname{eext}_{i, t, y, k}=0, \forall i \in I_{e}, t \in T_{\text {des }}, y \in Y, k \in K, E I N D_{i, t, y, k}=0 \\
& \operatorname{edis}_{i, t, y, k}=0, \forall i \in I_{e}, t \in T_{\text {des }}, y \in Y, k \in K, E I N D_{i, t, y, k}=0 \\
& \operatorname{eimp}_{s, t, t t}=0, \forall s \in S, t \in T_{o p t}, t t \in T_{r e p}, E I N D_{i, t, y, k}=0 \\
& \operatorname{eexp}_{s, t, t t}=0, \forall s \in S, t \in T_{\text {opt }}, t t \in T_{\text {rep }}, E I N D_{i, t, y, k}=0
\end{aligned}
$$

\section{Non-negativity and binary constraints}

The non-negativity constraints for the storage-related continuous decision variables are presented in constraints (62)-(66).

$$
\begin{aligned}
& \text { bact }_{i, t, y, t t} \geq 0, \forall i \in I_{b}, t \in T_{\text {opt }}, y \in Y, t t \in T_{\text {rep }} \\
& \text { bin }_{i, t, y, t t} \geq 0, \forall i \in I_{b}, t \in T_{\text {opt }}, y \in Y, t t \in T_{\text {rep }} \\
& \text { bout }_{i, t, y, t t} \geq 0, \forall i \in I_{b}, t \in T_{\text {opt }}, y \in Y, t t \in T_{\text {rep }} \\
& \text { bext }_{i, t, y} \geq 0, \forall i \in I_{b}, t \in T_{\text {des }}, y \in Y \\
& \text { bdis }_{i, t, y} \geq 0, \forall i \in I_{b}, t \in T_{\text {des }}, y \in Y
\end{aligned}
$$

Constraints (67)-(71) represent the non-negativity of power generation-related and importation-related continuous decision variables.

$$
\begin{aligned}
& \operatorname{egen}_{i, t, y, k, t t} \geq 0, \forall i \in I_{e}, t \in T_{\text {opt }}, y \in Y, k \in K, t t \in T_{\text {rep }} \\
& \operatorname{eext}_{i, t, y, k} \geq 0, \forall i \in I_{e}, t \in T_{\text {des }}, y \in Y, k \in K \\
& \operatorname{edis}_{i, t, y, k} \geq 0, \forall i \in I_{e}, t \in T_{\text {des }}, y \in Y, k \in K \\
& \operatorname{eimp}_{s, t, t t} \geq 0, \forall s \in S, t \in T_{\text {opt }}, t t \in T_{\text {rep }} \\
& \operatorname{eexp}_{s, t, t t} \geq 0, \forall s \in S, t \in T_{\text {opt }}, t t \in T_{\text {rep }}
\end{aligned}
$$

The binary decision variables are depicted by constraint (72).

$$
u_{i, t, y, k} \in\{0,1\}, \forall i \in I_{e}, t \in T_{\text {des }}, y \in Y, k \in K
$$




\section{Nomenclature}

\section{Sets/indices}

I

$I_{e}$

$I_{e i}$

$I_{\text {eni }}$

$I_{e r}$

$I_{e n r}$

$I_{b}$

K

S

T

$T_{\text {des }}$

$T_{\text {opt }}$

set of all technologies, indexed by $i$, including electricity generation technologies $\left(I_{e}\right)$ and electricity storage technologies $\left(I_{s}\right)$

set of electricity generation technologies, subset of $I$, including bituminous coal (BIT), fuel oil (FO), methane from biogas (MTE), refuse of solid waste (REF), utility solar PV (SUNU), distributed solar PV (SUND), nuclear power (UR), hydropower (WAT), on-land wind (WON), offshore wind (WOFF), natural gas combined cycle (NGCC), natural gas combustion turbine (NGGT), natural gas steam turbine (NGST), natural gas combined cycle with carbon capture and storage (NCCS)

set of intermittent renewable electricity generation technologies, subset of $I_{e}$, including utility solar PV (SUNU), distributed solar PV (SUND), on-land wind (WON), and offshore wind (WOFF)

set of non-intermittent renewable electricity generation technologies, subset of $I_{e}$, including methane from biogas (MTE), hydropower (WAT)

set of renewable electricity generation technologies, subset of $I_{e}$, including methane from biogas (MTE), hydropower (WAT), PV (SUNU), distributed solar PV (SUND), on-land wind (WON), and offshore wind (WOFF)

set of non-renewable electricity generation technologies, subset of $I_{e}$, including bituminous coal (BIT), fuel oil (FO), refuse of solid waste (REF), nuclear power (UR), natural gas combined cycle (NGCC), natural gas combustion turbine (NGGT), natural gas steam turbine (NGST), natural gas combined cycle with carbon capture and storage (NCCS)

set of electricity storage technologies, subset of $I$, including lithium-ion battery (LIB), flywheel (FW), and hydro (PSH)

set of generators or storage units with the same technology and the same age source of imported electricity, indexed by $s$ set of all time periods and time slices, indexed by $t$ and $t t$ set of time periods for capacity planning decisions, indexed by $t$ set of operational years within the planning horizon, indexed by $t$ 
$T_{\text {rep }} \quad$ set of time slices (hour) for electric power systems operational decisions, indexed by $t t$, start with $H I N I$, end with $H M A X$

$T_{\text {year }} \quad$ set of all years within the planning horizon, indexed by $t$

$Y \quad$ set of ages for all technologies, indexed by $y$, and $y=0$ indicates that the technology has just been deployed this year

\author{
Non-Negative Continuous variables

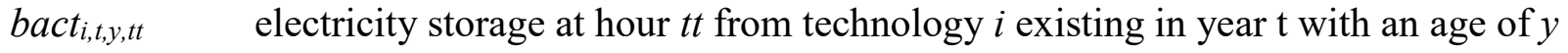 \\ bdisi,t,y electricity storage capacity of technology $i$ discarded in period $t$ with an age of $y$ \\ bext $_{i, t, y} \quad$ electricity storage capacity of technology $i$ existing in period $t$ with an age of $y$, and \\ $y=0$ indicates new units deployed in period $t$

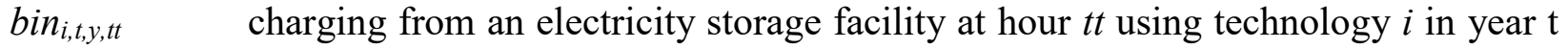 \\ with an age of $y$

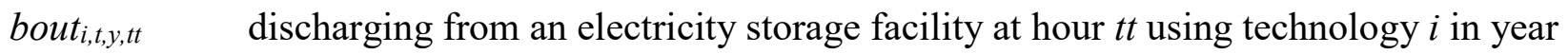 \\ $\mathrm{t}$ with an age of $y$ \\ cdes $_{i, t} \quad$ capacity planning cost, including investment cost and retirement/decommissioning \\ cost of technology $i$ in planning period $t$ \\ $\operatorname{cimp}_{s, t} \quad$ electricity importation cost from source $s$ in year $t$ \\ copt $_{i, t} \quad$ operational cost of technology $i$ in year $t$ \\ edis $s_{i, t, y, k} \quad$ electricity generation capacity of generator $k$ with technology $i$ decommissioned in \\ period $t$ with an age of $y$ \\ eext $t_{i, t, k, k} \quad$ electricity generation capacity from generator $k$ with technology $i$ existing in period \\ $\mathrm{t}$ with an age of $y$, and $y=0$ indicates new units deployed in period $t$

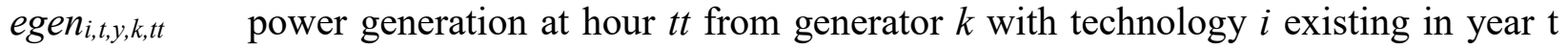 \\ with an age of $y$ \\ eimp $p_{s, t, t t} \quad$ electricity importation at hour $t t$ in year $y$ from source $s$ \\ tcd net present cost for capacity planning \\ tco net present cost for system operations \\ $z_{i, t, y, k, t t} \quad$ maximum available generation capacity considering the on/off status \\ Integer variables \\ $u_{i, t, y, k} \quad$ decommissioning of generator $k$ with technology $i$ in year $\mathrm{t}$ with an age of $y$
}

\title{
Parameters
}


$A V R E_{i, t} \quad$ annual availability for variable renewable energy for technology $i$ in year $t$

$B F_{i, t, y} \quad$ energy storage factor for unit storage capacity using technology $i$ in year $t$ with an age of $y$

$B I N D_{i, t, y} \quad$ indicator of active energy storage unit using technology $i$ in year $t$ with an age of $y$

$B L O S_{i, t, y} \quad$ hourly energy loss in storage facilities for technology $i$ in year $t$ with an age of $y$

$C C A R_{t} \quad$ estimated social cost for carbon dioxide emissions in year $t$

$C C A P_{i, t, y, k} \quad$ unit capital cost for the construction of a new unit of technology $i$ in year $t$, equals zero when $y \neq 0$

$C D E P_{i, t, y} \quad$ cost due to early retirement for units using technology $i$ in year $t$ with an age of $y$

$C F_{i, t, y, t t} \quad$ capacity factor for intermittent electricity generation technology $i$ at hour $t t$ in year $t$ with an age of $y$

CFIX $X_{i, t, y} \quad$ fixed operations and maintenance cost on unit power capacity of electricity generation technology $i$ in year $t$ with an age of $y$

$C I M P_{s, t, t t} \quad$ cost associated with unit amount of imported electricity from source $s$ in year $t$ at hour $t t$

$C O P T_{i, t, y} \quad$ annual unit operations and maintenance cost for storage technology $i$ in year $t$ with an age of $y$

$C S A L_{i, t} \quad$ decommissioning cost for retired unit $k$ using technology $i$ in year $t$ at the end of its useful life, associated with unit amount of annual energy production

$C V A R_{i, t, y, t t} \quad$ variable operations and maintenance cost on unit power generation of electricity generation technology $i$ in year $t$ with an age of $y$ at hour $t t$

$D E M E_{t, t t} \quad$ electricity demand in year $t$ at hour $t t$

$E A N N_{t} \quad$ annual demand for electricity generation in year $t$

$E E_{i, t, y} \quad$ greenhouse gas emissions regarding unit amount of electricity generated from technology $i$ in year $t$ with an age of $y$

$E I N D_{i, t, y, k} \quad$ indicator of active generator $k$ using technology $i$ in year $t$ with an age of $y$

$E L M T_{t} \quad$ limit for annual greenhouse gas emission in year $t$

$G R O B_{i, t} \quad$ maximum increase of electricity storage capacity of technology $i$ in year $t$

$G R O E_{i, t} \quad$ maximum increase of generation capacity using technology $i$ in year $t$

$H O U R_{i, t, y} \quad$ working hours for technology $i$ in year $t$ with an age of $y$

$I M P M_{s, t} \quad$ maximum electricity importation capacity from source $s$ in year $t$ 
$I N I E_{i, y, k} \quad$ electricity generation capacity for generator $k$ using technology $i$ with an age of $y$ in year TINI

INIB $\quad$ storage capacity of technology $i$ with an age of $y$ in year TINI

$L B C_{i} \quad$ lower bound for capacity of a generator using technology $i$

$L C A P_{i, t, y} \quad$ capacity limit for single unit $k$ with technology $i$ in year $t$ with an age of $y$

$L_{i F E_{i}} \quad$ lifetime for technology $i$

$M$

a large positive number applied for the big-M method

$M S_{i, t}$

$N H I_{i, y}$

upper bound for the total market share (percentages) of technology $i$ in year $t$

$N L O_{i, y}$ maximum increase percentage for new deployments using technology $i$ in year $y$

$P R O B_{i, t}$ maximum reduction percentage for new deployments using technology $i$ in year $y$

$P R O D_{i, t, y, k}$ proposed energy storage capacity additions with technology $i$ in year $t$

$P R O G_{i, t, k} \quad$ proposed capacity additions of generator $k$ with technology $i$ in year $t$

RATE discount rate

$R E D B_{i, t} \quad$ maximum reduction of electricity storage capacity of technology $i$ in year $t$

$R E D E_{i, t} \quad$ maximum reduction of generation capacity using technology $i$ in year $t$

$R E P E_{t} \quad$ total electricity demand for all time slices in year $t$

$R_{P S G_{t}} \quad$ renewable portfolio standards on renewable generation for the electric power sector in year $t$, determined by the government

$T A R_{i, t} \quad$ target capacity level for technology $i$ in year $t$

$T A R S_{t} \quad$ target capacity level for electricity storage in year $t$

TINI initial year for the planning horizon

TMAX planning time horizon in years

$T R E_{t} \quad$ total generation capacity changes in percentage

$T R S_{t} \quad$ total energy storage capacity changes in percentage

$U B C_{i} \quad$ upper bound for capacity of a generator using technology $i$

a small positive number 